\title{
Prevalencia de la infección con el virus linfotrópico de células T humanas de tipo 1 y 2 en donantes de sangre en Colombia, 2001-2014: implicaciones sobre la seguridad de la transfusión
}

\author{
María Isabel Bermúdez-Forero, Maritza Berrío-Pérez, Andrea Magally Herrera-Hernández, \\ Magda Juliana Rodríguez-Rodríguez, Sandra García-Blanco, \\ Guillermo Orjuela-Falla, Mauricio Beltrán \\ Coordinación de la Red Nacional de Bancos de Sangre y Servicios de Transfusión, \\ Instituto Nacional de Salud, Bogotá D.C., Colombia
}

Introducción. El virus linfotrópico de células T humanas (HTLV) de tipos 1 y 2 ocasiona trastornos clínicos asociados a enfermedades degenerativas y proliferativas. Entre sus principales mecanismos de transmisión está la transfusión, asociada principalmente al uso de componentes celulares como los glóbulos rojos.

Objetivo. Describir la epidemiología del HTLV 1 y 2 en donantes de sangre en Colombia, entre 2001 y 2014.

Materiales y métodos. Se hizo un análisis descriptivo y retrospectivo de la información enviada por la Red de Bancos de Sangre al Instituto Nacional de Salud sobre tamización, unidades reactivas y positividad para el HTLV 1 y 2 y sobre la estimación de riesgo de infección por la transfusión.

Resultados. Entre 2001 y 2014 se hizo en Colombia la tamización para la detección de anticuerpos de HTLV 1 y 2 de 60,2 \% de la sangre captada, con una tasa acumulada de unidades reactivas de 0,3\%. Dicha tasa fue 20 veces superior en el departamento de Chocó (6,28\%), pese a que allí no se capta sangre desde el 2004. En el 2014, la tamización llegó a 94,9 \%, con una positividad de 14,7 \%. Con estos datos se pudo estimar que se transfundieron 406 unidades de glóbulos rojos potencialmente infecciosos, lo cual entrañaría una transmisión eficaz del virus a estos individuos. Pese a que no se le considera un departamento endémico, en Antioquia se registró la mayor proporción de pruebas positivas, con 215 unidades (53\%).

Conclusiones. Los resultados obtenidos sugieren que la infección por HTLV 1 y 2 se distribuye en varias zonas del país que no eran catalogadas como endémicas. Se ratificó la importancia de la tamización universal de las unidades de sangre captadas, para minimizar el riesgo de infección con este agente por la vía de la transfusión.

Palabras clave: virus 1 linfotrópico T humano, transfusión sanguínea, bancos de sangre, donantes de sangre, prevalencia, Colombia.

doi: http://dx.doi.org/10.7705/biomedica.v36i0.2943

Prevalence of human T-cell lymphotropic virus I and II in Colombian blood donors, 2001-2014: Implications for transfusion safety

Introduction: The human T-cell lymphotropic virus (HTLV) 1 and 2 cause various clinical disorders associated with degenerative diseases. Blood transfusion is a primary mechanism of transmission that is associated with the use of cellular components such as red blood cells.

Objective: To describe the epidemiology of HTLV 1 and 2 in blood donors in Colombia from 2001-2014. Materials and methods: A retrospective analysis was performed using screening, reactivity and positivity for HTLV 1 and 2 data collected from 2001 to 2014 by Colombian blood banks and consolidated by the Instituto Nacional de Salud. Using this information, transfusion-associated infectivity was also estimated.

Results: From 2001 to $2014,60.2 \%$ of blood collected in Colombia was screened for HTLV 1 and 2 and had a cumulative reactivity of $0.30 \%$. This was 20 times higher in Chocó (6.28\%), where blood collection

\section{Contribución de los autores:}

María Isabel Bermúdez-Forero: concepción y diseño, consolidación, análisis e interpretación de los datos Maritza Berrío-Pérez: consolidación, análisis e interpretación de los datos

Andrea Magally Herrera-Hernández, Magda Juliana Rodríguez-Rodríguez, Sandra Milena García-Blanco: marco conceptual

Guillermo Orjuela-Falla y Mauricio Beltrán: marco conceptual y apoyo en el análisis de los datos

Todos los autores participaron en la revisión crítica del documento y en la escritura del manuscrito. 
ended in 2004. Blood screening for HTLV reached $94.9 \%$ in 2014 with a positive concordance of $14.7 \%$, and an estimated 406 unscreened, potentially infectious blood units were released. The majority of the unscreened blood units (215 units, 53\%) came from Antioquia, a non-endemic department.

Conclusion: These results suggest that HTLV 1 and 2 infections are distributed in different areas of the country that were not previously classified as endemic. These findings support the importance of the universal screening of blood units to minimize the risk of infection through transfusion for this event.

Key words: Human T-lymphotropic virus 1, blood transfusion, blood banks, blood donors, prevalence, Colombia.

doi: http://dx.doi.org/10.7705/biomedica.v36i0.2943

El virus linfotrópico de células T humanas (HTLV) se transmite por vía vertical, sexual y parenteral, por lo que ha sido reportado en usuarios de drogas intravenosas y en pacientes transfundidos. Se ha encontrado que puede permanecer latente por periodos prolongados en zonas endémicas y grupos familiares (1). Los portadores del HTLV-1 pueden desarrollar enfermedades asociadas a la infección a lo largo de sus vidas, como la leucemia de células $T$ del adulto (2-4\% de los infectados) y la paraparesia espástica tropical $(1,5-3 \%$ de los infectados) $(1,2)$.

El HTLV-1 se ha estudiado ampliamente, pero a pesar de ello, aún no se tiene una cifra aproximada del número de individuos infectados en el mundo (3) y la mayoría de los datos de prevalencia provienen de estudios de donantes de sangre de bajo riesgo o en grupos no representativos seleccionados de la población general (4-7).

En un estudio publicado en 1996, se estimó que en el mundo había entre 15 y 20 millones de personas con el HTLV-1 (4); en Suramérica, la infección por el HTLV-1 se ha reportado en la mayoría de los países (3).

EI HTLV-I fue detectado en Colombia en 1981 por Zaninovic, et al., en una serie de pacientes provenientes de algunas poblaciones de la costa Pacífica que padecían de paraparesia espástica (8). El mismo grupo documentó sistemáticamente los reportes de caso y la prevalencia de la infección en otras regiones y grupos poblacionales del país, especialmente comunidades indígenas $(9,10)$. En un estudio de una muestra de individuos

\footnotetext{
Correspondencia:

María Isabel Bermúdez-Forero, Coordinación Red Nacional de Bancos de Sangre y Servicios de Transfusión, Instituto Nacional de Salud, Avenida Calle 26 № 51-20, Bogotá D.C., Colombia Teléfono: (571) 2212219 y 220 7700, extensión 1254 o 1255 mbermudez@ins.gov.co

Recibido: 13/07/15; aceptado: 03/05/16
}

provenientes de una zona endémica (Tumaco, Nariño), se encontraron tasas de prevalencia de anticuerpos contra el HTLV-1 cercanas a 3 \% (11).

En trabajos más recientes, se evidenció la dificultad del diagnóstico clínico, incluso en zonas consideradas de alta endemia (12). Debido a la lenta progresión de los síntomas y su asociación con otras alteraciones neurológicas, los casos de paraparesia representan un reto para el diagnóstico (4). Por otro lado, Carrascal, et al., adelantaron un estudio enfocado en la detección del virus en posibles casos no diagnosticados de leucemia o linfoma de células T en una zona endémica, y encontraron que 5 de 75 pacientes con linfoma no Hodgkin eran positivos para la secuencia del HTLV-I mediante reacción en cadena de la polimerasa (PCR) (13).

En un estudio reciente en Colombia, se describieron dos casos clínicos de pacientes procedentes de una zona endémica que padecían de leucemia - linfoma de células $T$ y que solo pudieron ser diagnosticados en un servicio de patología de alta complejidad (14).

Un amplio número de investigaciones se han enfocado en donantes voluntarios de sangre para aproximarse al estudio de la presencia del virus en una región determinada. Dada su capacidad de transmitirse por medio de la transfusión, la detección de la infección en donantes de sangre ha resultado de enorme impacto en salud pública (15).

La tasa de infectividad (infectivity rate) establecida a partir de unidades de sangre seropositivas varía, con un promedio cercano a $27 \%$ (16), aunque en algunos reportes se sostiene que las tasas pueden oscilar entre 20 y $63 \%$ (17). A pesar de que no se ha demostrado que la leucorreducción prevenga por completo la transmisión, algunos estudios evidencian que la mitiga en grados variables (18). Se estima que, en ausencia de la prueba de tamización o de la leucorreducción, el riesgo de transmisión sería de alrededor de dos a 63 por millón de habitantes en países desarrollados, 
donde la prevalencia de la infección oscila entre uno por 10.000 a uno por 100.000 . Este riesgo se ha reducido mucho (más de $95 \%$ ) gracias a la introducción de la prueba serológica de tamización en donantes. El riesgo calculado para los Estados Unidos es de aproximadamente uno en tres millones de unidades $(18,19)$.

Es importante resaltar que en Colombia el proceso de confirmación, notificación, asesoría y canalización de donantes de sangre positivos para los marcadores infecciosos relevantes comenzó a implementarse en los bancos de sangre con la expedición de la circular 082 de 2011 (20). A partir de febrero de 2014 las pruebas de detección del HTLV 1 y 2 son obligatorias para todos los donantes de sangre del país (21). Por esta razón, se planteó hacer un estudio retrospectivo de la información remitida a la red de bancos de sangre que permitiera describir la epidemiología del HTLV en donantes de sangre de Colombia desde 2001, año en el cual se inició la tamización para HTLV 1 y 2, hasta 2014, último año en el cual la tamización fue inferior a $100 \%$.

\section{Materiales y métodos}

Se hizo un análisis descriptivo retrospectivo con base en la información consolidada por la Red Nacional de Bancos de Sangre y Servicios de Transfusión del Instituto Nacional de Salud desde 2001 y hasta 2014.

En los análisis de las variables cuantitativas se incluyeron las unidades de sangre obtenidas, las sometidas a tamización, las reactivas (es decir, con resultado positivo en la prueba de tamización) y las positivas (resultado confirmado mediante pruebas de inmunoblot).

Se asumió que las unidades no sometidas a tamización presentaban un comportamiento similar a las tamizadas con respecto al número de unidades reactivas y la proporción de casos positivos confirmados. Teniendo en cuenta que el proceso de confirmación sistemática de las unidades reactivas en los bancos de sangre se viene implementando desde mediados de 2012, pero que solo en el 2014 se logró la unificación y la depuración de los datos, se tomó como referencia el porcentaje de positividad obtenido para este último año en las estimaciones hechas en el periodo de estudio.

Se estimó el riesgo de infección discriminado por departamento y por año empleando para el cálculo una tasa de infección de $27 \%$ en promedio para las unidades positivas confirmadas, según lo establecido en la literatura científica (22).
Para las estimaciones se consideró que solo se había transfundido un componente sanguíneo por cada unidad de sangre total recolectada.

\section{Resultados}

En el periodo comprendido entre 2001 y 2014 , se captaron 8'478.364 unidades de sangre total en todo el país, de las cuales 60,2 \% (5'105.159) se sometió a tamización para HTLV 1 y 2 y $0,3 \%$ (15.480) resultaron reactivas.

La tamización pasó de 15,3 \% en 2001 a 75,4 \% en 2013, pese a que el escrutinio de este marcador solo se hizo obligatorio en 2014, cuando llegó a $94,9 \%$.

Los departamentos en los que se hizo tamización para HTLV de más de $90 \%$ fueron Valle del Cauca, Nariño y Caldas. En el departamento del Chocó se hizo tamización en $19,3 \%$ de las unidades de sangre obtenidas y $6,28 \%$ de la unidades fueron reactivas, es decir, 20 veces superior al promedio nacional $(0,30 \%)$ durante el periodo estudiado, pese a que este departamento no capta sangre desde el 2004. En los departamentos de Córdoba, Cauca, Antioquia, Caldas, Casanare y Magdalena ese promedio fue entre 1,7 y 3,1 mayor que el nacional (cuadro 1).

Los departamentos que tamizaron menos de $10 \%$ de las unidades obtenidas fueron Caquetá, La Guajira, Casanare y Sucre, a pesar de que estos dos últimos presentaron un promedio de unidades reactivas superior al promedio nacional $(0,75 \mathrm{y}$ $0,96 \%$, respectivamente).

Al agrupar los resultados de las unidades reactivas por regiones, el mayor porcentaje se observó en la región Pacifica $(7,52 \%)$, seguida de la Caribe $(3,53 \%)$, la Andina (3,08\%), la Orinoquia (1,27\%), y la Amazonia (0,20\%).

Al aplicar la misma proporción de unidades reactivas y de positividad de las unidades tamizadas, se calculó que 1.504 unidades de los 3'373.205 no tamizadas podrían haber sido positivas $(0,045 \%)$. Debido a la ausencia de una prueba de tamización universal para HTLV entre el 2001 y el 2014 en Colombia, se estimó que se transfundieron 406 unidades de glóbulos rojos empaquetados con capacidad de transmisión eficiente del virus HTLV 1 y 2 a los receptores (figura 1).

Al considerar el volumen de captación, el departamento que presentó el mayor riesgo de capacidad infecciosa fue Antioquia, en donde se calculó que $215(53 \%)$ unidades estaban infectadas, seguido de Atlántico, con 48 unidades (11,8\%) (cuadro 1). 
Cuadro 1. Comportamiento de la tamización, las unidades reactivas y la probabilidad de infección del HTLV 1 y 2 por departamento, Colombia, 2001-2014

\begin{tabular}{|c|c|c|c|c|c|}
\hline Departamento o distrito & $\begin{array}{l}\text { Unidades de sangre } \\
\text { obtenidas (2001 - 2014) }\end{array}$ & $\begin{array}{c}\text { Unidades } \\
\text { tamizadas (\%) }\end{array}$ & $\begin{array}{c}\text { Unidades } \\
\text { reactivas (\%) }\end{array}$ & $\begin{array}{l}\text { Unidades } \\
\text { positivas }\end{array}$ & $\begin{array}{l}\text { Probabilidad } \\
\text { de infección }\end{array}$ \\
\hline Antioquia & 1’296.386 & 23,1 & 0,54 & 797 & 215 \\
\hline Atlántico & 426.332 & 13,2 & 0,33 & 178 & 48 \\
\hline Bogotá, D.C. & 2’556.081 & 84,3 & 0,21 & 124 & 33 \\
\hline Bolívar & 196.509 & 32,8 & 0,32 & 63 & 17 \\
\hline Boyacá & 129.738 & 65,5 & 0,18 & 12 & 3 \\
\hline Caldas & 210.553 & 93,7 & 0,57 & 11 & 3 \\
\hline Caquetá & 35.084 & 2,8 & 0,20 & 10 & 3 \\
\hline Cauca & 83.908 & 59,4 & 0,54 & 27 & 7 \\
\hline Cesar & 171.233 & 71,2 & 0,47 & 34 & 9 \\
\hline Córdoba & 206.247 & 51,9 & 0,50 & 73 & 20 \\
\hline Cundinamarca & 224.443 & 86,1 & 0,37 & 17 & 5 \\
\hline Chocó & 1.979 & 19,3 & 6,28 & 15 & 4 \\
\hline Huila & 164.722 & 17,6 & 0,20 & 39 & 11 \\
\hline La Guajira & 41.922 & 7,0 & 0,00 & 0 & 0 \\
\hline Magdalena & 83.305 & 14,5 & 0,94 & 99 & 27 \\
\hline Meta & 116.548 & 75,1 & 0,41 & 17 & 5 \\
\hline Nariño & 114.457 & 98,4 & 0,35 & 1 & 0 \\
\hline Norte de Santander & 167.649 & 16,8 & 0,28 & 57 & 15 \\
\hline Quindío & 109.063 & 67,0 & 0,18 & 9 & 3 \\
\hline Risaralda & 168.751 & 64,3 & 0,34 & 30 & 8 \\
\hline Santander & 513.955 & 19,3 & 0,18 & 111 & 30 \\
\hline Sucre & 91.350 & 4,8 & 0,96 & 123 & 33 \\
\hline Tolima & 438.049 & 80,4 & 0,25 & 31 & 8 \\
\hline Valle del Cauca & 859.654 & 99,8 & 0,35 & 1 & 0 \\
\hline Arauca & 26.514 & 16,7 & 0,11 & 4 & 1 \\
\hline Casanare & 30.903 & 8,7 & 0,75 & 31 & 8 \\
\hline Putumayo & 9.154 & 0,0 & -- & -- & -- \\
\hline Guainía & 257 & 100,0 & -- & -- & -- \\
\hline Guaviare & 603 & 0,0 & -- & -- & -- \\
\hline Vichada & 2.945 & 0,0 & -- & -- & -- \\
\hline Total nacional & 8'478.364 & 60,2 & 0,30 & 1.503 & 406 \\
\hline
\end{tabular}

-- No fue posible estimar el riesgo de infección por no contar con los datos de tamización o de unidades reactivas.

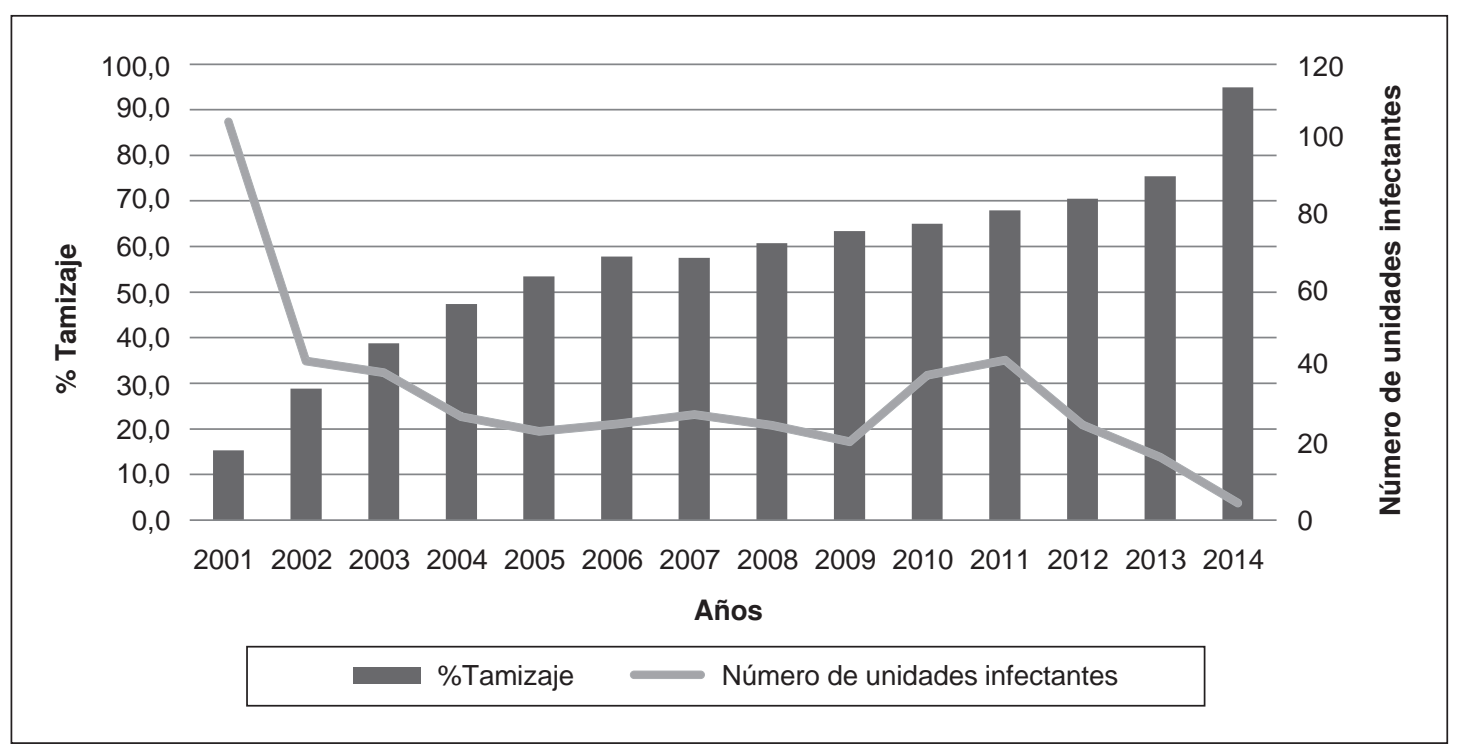

Figura 1. Comparación del comportamiento del porcentaje de tamización y el número estimado de unidades infecciosas liberadas, Colombia, 2001-2014 
Con el incremento paulatino de la tamización se observó reducción de unidades potencialmente infectante, al pasar de 105 en 2001 a cinco unidades en 2014.

Los departamentos con menos unidades reactivas, los cuales aportaron $22,6 \%$ de las unidades infecciosas que se habrían distribuido, fueron Arauca, Quindío, Boyacá, Huila, Caquetá, Bogotá, Santander y Tolima; en estos tres últimos, se captó $41,4 \%$ de las unidades de sangre obtenidas en el periodo de estudio.

En Guainía, Guaviare, La Guajira, Putumayo y Vichada, no fue posible establecer el riesgo de infección porque ninguna de las unidades obtenidas fue tamizada o porque de las unidades tamizadas ninguna resultó reactiva durante el periodo analizado.

\section{Discusión}

Pese a que la distribución de las unidades reactivas para el HTLV pudo verse sesgada por el porcentaje de unidades de sangre tamizadas, se observó que, tal como lo describen los estudios poblacionales, en las regiones Pacífica y Caribe de Colombia se concentraron las unidades reactivas. Sin embargo, se registró una gran 'reactividad' en los departamentos que conforman la región Andina, aun cuando esta región no se considera endémica para este virus. Los cambios en la prevalencia de diversos agentes infecciosos en varias zonas del país podrían explicarse por la migración de la población.

Aunque el porcentaje de unidades reactivas fue el indicador inicial a partir del cual se estimó el riesgo de capacidad infecciosa, y la mayor 'reactividad' se registró en el Chocó, Antioquia aportó $53 \%$ de las unidades potencialmente infecciosas (215/ 406) durante el periodo analizado, lo cual podría deberse al volumen de captación de este último departamento comparado con el registrado a nivel nacional (15,3\%).

Se observó un descenso en la prevalencia de donantes de sangre $(0,09 \%)$ comparado con los resultados del estudio en esta misma población publicado en 1999, el cual se llevó a cabo en bancos de sangre del país situados en zonas tanto endémicas como no endémicas, donde la prevalencia promedio fue de 0,45\% (23). La relación entre tamización y unidades reactivas presentó un comportamiento inversamente proporcional, lo cual puede atribuirse, entre otras razones, a una mejoría del proceso de selección de donantes o a una mayor especificidad de las pruebas de tamización.
Estos hallazgos demuestran la importancia de implementar la tamización universal para el HTLV 1 y 2, considerando la distribución de la 'reactividad' en el territorio nacional. Además, si se analizan los datos de departamentos como Antioquia en el contexto de su estatus de referente por el nivel de complejidad de su red hospitalaria, se hace más crítico el contar con el mayor número de estrategias que minimicen los riesgos para los pacientes que requieren transfusión.

Teniendo en cuenta que uno de los principales mecanismos de trasmisión del HTLV 1 y 2 son las trasfusiones, a partir de 2014 se estableció la obligatoriedad de la tamización del total de las unidades de sangre captadas, con lo que se espera reducir la trasmisión y minimizar el riesgo de liberar unidades que puedan generar la infección. Asimismo, reviste especial importancia el hecho de que los bancos de sangre actualmente brindan asesoría a los donantes que resultan positivos, dado que la consejería médica a un portador asintomático es un reto enorme, sobre todo por el gran desconocimiento de la enfermedad entre el personal médico de atención primaria. Por ello, el contar con la información adecuada puede incentivar a esta población a acudir a sus servicios de salud de manera temprana y a implementar prácticas que minimicen la transmisión por las demás vías clásicas (materna y sexual).

Al comparar los resultados obtenidos en las pruebas de tamización durante los 13 años del periodo analizado, se pudo estimar una tasa de 303 unidades reactivas por cada 100.000 tamizadas, lo cual contrasta con el estudio de Carneiro, et al., en una población de donantes de primera vez en Brasil entre 2007 y 2009, en el cual se reportó una tasa de 135 unidades reactivas por 100.000 unidades tamizadas (24).

La tasa nacional de positividad calculada para el periodo de 2001 a 2014 (45 donaciones positivas por cada 100.000 efectuadas), contrasta con lo publicado para el periodo de 2000 a 2009 en donantes de primera vez en Estados Unidos, donde se halló una tasa de 5,1 por 100.000 unidades para el HTLV 1 y de 14,7 por 100.000 para el HTLV 2 (25).

Cuando se constata que la tasa calculada en Colombia es comparable con la de Brasil, país considerado endémico para el virus, cobra mayor relevancia la investigación poblacional (24).

Aunque el riesgo de producir infección de las 406 unidades de glóbulos rojos con potencial infeccioso dependía de diversas variables, un 
número importante de pacientes podría desarrollar en el futuro las enfermedades que este virus provoca, para las cuales, como se sabe, no existen tratamientos curativos. Esto tendría un impacto considerable en el sistema de salud pública del país, debido a las implicaciones del tratamiento paliativo y los años de calidad de vida perdidos de los pacientes que las desarrollen. Aunque se ha encontrado que solo un porcentaje de los portadores asintomáticos llegan a desarrollar alguna de las enfermedades asociadas al virus, es importante considerar que estas no son de fácil diagnóstico y que en la actualidad no tienen cura, por lo que su tratamiento consiste en la administración de paliativos que procuran mantener la calidad de vida de los pacientes. Por consiguiente, si se busca minimizar la morbilidad asociada, es importante conocer la distribución endémica actual del virus, con el fin de orientar las estrategias de prevención que eviten al máximo su proliferación en la población colombiana.

Aunque los datos obtenidos en donantes de sangre representan un grupo de población preseleccionado, el cual se considera de bajo riesgo para infecciones transmisibles por vía parenteral, es posible extrapolar esta información para que constituya la línea de base de un eventual estudio sobre el comportamiento y la distribución regional del HTLV en la población general, en la cual la prevalencia podría ser superior.

Los resultados obtenidos evidenciaron la presencia del HTLV 1 y el 2 en diferentes zonas del país previamente catalogadas como no endémicas. Dada la importancia de este virus y a sus mecanismos de trasmisión, los estudios poblacionales cobran relevancia para conocer su distribución actual en la población colombiana, y para contar con datos más precisos sobre la incidencia y la prevalencia de la infección por HTLV 1 y 2 . Los resultados de tales estudios permitirían considerar su inclusión como una situación de interés en salud pública debido a las enfermedades que puede desencadenar en el paciente infectado.

Los resultados de este estudio ratifican la importancia de la tamización universal para el HTLV de las unidades de sangre captadas, con el fin de minimizar los riesgos de infección por la vía de la transfusión.

\section{Agradecimientos}

Al personal de los bancos de sangre y a las coordinaciones departamentales de la Red Nacional de Bancos de Sangre del país.

\section{Financiación}

Este trabajo se realizó con el apoyo financiero del Instituto Nacional de Salud.

\section{Conflicto de intereses}

Ninguno

\section{Referencias}

1. Romaní F. Revisión sistemática de estudios epidemiológicos sobre la infección por el virus linfotrópico de células $T$ humanas I/II en el Perú. Revista Peruana de Epidemiología. 2010;14:1-9.

2. Dow BC, Fiebig EW, Busch MP. Retroviruses. En: Barbara JA, Regan FA, Contreras M, editors. Transfusion Microbiology. First edition. Cambridge: Cambridge University Press; 2008. p. 63.

3. Villaverde JA, Romaní FR, Torres SM, Zunt JR. Transmisión vertical de HTLV-1 en el Perú. Rev Peru Med Exp Salud Pública. 2011;28:101-8. http://dx.doi.org/10.1590/ S1726-46342011000100016

4. Proietti FA, Carneiro-Proietti AB, Catalan-Soares BC, Murphy EL. Global epidemiology of HTLV-I infection and associated diseases. Oncogene. 2005;24:6058-68. http:// dx.doi.org/10.1038/sj.onc. 1208968

5. Hlela C, Shepperd S, Khumalo NP, Taylor GP. The prevalence of human T-cell lymphotropic virus type 1 in the general population in unknown. AIDS Rev. 2009;11:205-14.

6. Gotuzzo E. Risk of transfusion-transmitted human T-cell lymphotropic virus-type I in Latin America. Int J Infect Dis. 2000;4:59-61. http://dx.doi.org/10.1016/S1201-9712(00) 90094-8

7. Gotuzzo E, Verdonck K, González E, Cabada M. Virus linfotrópico humano de células T tipo 1 (HTLV-1): una infección endémica en el Perú. Rev Peru Med Exp Salud Pública. 2004;21:253-60.

8. Zaninovic V, Arango C, Biojó R, Mora C, RodgersJohnson P, Concha M, et al. Tropical spastic paraparesis in Colombia. Ann Neurol. 1988;23(Supl.1):127-32.

9. Zamora T, Zaninovic V, Kajiwara M, Komoda H, Hayami M, Tajima K. Antibody to HTLV-I in indigenous inhabitants of the Andes and Amazon regions in Colombia. Jpn $\mathrm{J}$ Cancer Res. 1990;81:715-9.

10. Zaninovic V, Moreno D. A propósito de cinco casos de paraparesia espástica tropical en Puerto Tejada (Cauca). Colombia Médica. 1997;28:67-70.

11. Trujillo J, Concha M, Muñoz A, Bergonzoli G, Mora C, Borrero I. Seroprevalence and cofactors of HTLV-I infection in Tumaco, Colombia. AIDS Res Hum Retroviruses. 1992;8:651-7. http://dx.doi.org/10.1089/aid.1992.8.651

12. Rosero F, Aguirre C, Rosero M, Orjuela DL, Rosero A. Paraparesia espástica tropical en un paciente con HTLV-I. Neurología Argentina. 2011;3:229-33. http://dx.doi. org/10.1016/j.neuarg.2011.04.003

13. Carrascal E, Cortés A, Akiba S, Tamayo O, Quiñónez F. Epidemiología y patología de la leucemia/linfoma de las células T del adulto en Cali y el suroccidente colombiano. Colombia Médica. 2004;35:12-7. 
14. Medina EA, Orduz R, Morales OL, Martínez Ó, Baldión M, Isaza MA. Leucemia/linfoma T del adulto en pacientes infectados con HTLV-1: reporte de dos casos de Colombia. Biomédica. 2013;33:519-25. http://dx.doi.org/10.7705/ biomedica.v33i4.1429

15. Di Lorenzo-Oliveira C, Loureiro F, De Bastos MR, Proietti FA, Carneiro-Proietti AB. Blood donor deferral in Minas Gerais State, Brazil: Blood centers as sentinels of urban population health. Transfusion. 2009;49:851-7. http://dx.doi. org/10.1111/j.1537-2995.2008.02062.x

16. Whyte G. Is screening of Australian blood donors for HTLV-I necessary? Med J Aust. 1997;166:478-81.

17. Vrielink H, Reesink H. HTLV-I/II prevalence in different geographic locations. Transfus Med Rev. 2004;18:46-57. http://dx.doi.org/10.1016/j.tmrv.2003.10.004

18. Hewitt PE, Davison K, Howell DR, Taylor GP. Human T-lymphotropic virus lookback in NHS Blood and Transplant (England) reveals the efficacy of leukoreduction. Transfusion. 2013;53:2168-75. http://dx.doi.org/10.1111/trf.12105

19. Pomper G, Wu Y, Snyder E. Risks of transfusion-transmitted infections: 2003. Curr Opin Hematol. 2003;10:412-8.

20. Instituto Nacional de Salud. Circular No. 0082 de 2011. Pruebas confirmatorias de donantes de sangre serorreactivos. Fecha de consulta: 23 de mayo de 2015. Disponible en: http://www.ins.gov.co/lineas-de-accion/Red-NacionalLaboratorios/Normatividad\%20Red\%20nal\%20bancos\%20 de\%20sangre/Circular\%200082\%20de\%202011.pdf.
21. Ministerio de Salud y Protección Social. Resolución 437 de 2014. Fecha de consulta: 23 de mayo de 2015. Disponible en: https://www.minsalud.gov.co/Normatividad_ Nuevo/Resoluci\%C3\%B3n\%200437\%20de\%202014.pdf-

22. Manns A, Wilks RJ, Murphy EL, Haynes G, Figueroa JP, Barnett M, et al. A prospective study of transmission by transfusion of HTLV-I and risk factors associated with seroconversion. Int J Cancer. 1992;51:886-91.

23. Cortés A, Beltrán M, Gallego GA, Isaza LM. Estudio prospectivo seroepidemiológico de infección por el virus linfotrópico humano I y II (HTLV-I/II) en donantes de sangre de áreas colombianas endémicas y no endémicas. Colombia Médica.1999;30:19-25.

24. Carneiro-Proietti AB, Sabino EC, Leão S, Salles NA, Loureiro P, Sarr M, et al. Human T-lymphotropic virus type 1 and type 2 seroprevalence, incidence, and residual transfusion risk among blood donors in Brazil during 20072009. AIDS Res Hum Retroviruses. 2012;28:1265-72. http:// doi.org/10.1089/aid.2011.0143

25. Chang YB, Kaidarova Z, Hindes D, Bravo M, Kiely $\mathbf{N}$, Kamel $\mathbf{H}$, et al. Seroprevalence and demographic determinants of human T-lymphotropic virus Type 1 and 2 infections among first-time blood donors-United States, 2000-2009. J Infect Dis. 2014;209:523-31. http://doi.org/10. 1093/infdis/jit497 